\title{
APPROXIMATION PROPERTIES OF BASKAKOV-BALAZS TYPE OPERATORS FOR FUNCTIONS OF TWO VARIABLES
}

\author{
ÇiĞDEM ATAKUT, İBRAHIMM BÜYÜKYAZICI, AND SEVILAY K. SERENBAY
}

Received 28 February, 2014

\begin{abstract}
In this paper, we study Baskakov type positive operators in polynomial weighted spaces of functions of two variables. We obtain some well known operators by using our operators which are special cases of them. We give theorems on approximation, on the degrees of approximations of functions and the Vornovskaya type theorem for these operators. Finally, we present an open problem concerning the $q$ analogue of these operators.
\end{abstract}

2010 Mathematics Subject Classification: 41A25; 41A36

Keywords: Szász-Mirakjan operator, Balazs operator, Baskakov type operator, degree of approximation, Voronovskaya type theorem

\section{INTRODUCTION}

Let $p \in \mathbb{N}_{0}$. We define $\omega_{p}(u)=\left(1+u^{p}\right)^{-1}, u \in \mathbb{R}_{0}=[0, \infty)$ and for fixed $p, q \in$ $\mathbb{N}_{0}$, we define the weighted function

$$
\omega_{p, q}(x, y)=\omega_{p}(x) \omega_{q}(y), \quad(x, y) \in \mathbb{R}_{0}^{2}=[0, \infty) \times[0, \infty) .
$$

We denote by the weighted space $C_{\omega_{p, q}}$ the space of all real-valued functions $f$ continuous on $\mathbb{R}_{0}^{2}$ for which $\omega_{p, q} f$ is uniformly continuous and bounded on $\mathbb{R}_{0}^{2}$ with the norm

$$
\|f\|_{\omega_{p, q}}=\sup _{(x, y) \in \mathbb{R}_{0}^{2}} \omega_{p, q}(x, y)|f(x, y)| .
$$

The modulus of continuity of $f \in C_{\omega_{p, q}}$ defined by

$$
\Omega(f ; t, s)=\sup _{0 \leq h \leq t} \sup _{0 \leq \delta \leq s}\left\|\Delta_{h, \delta} f\right\|_{\omega_{p, q}}, t, s \geq 0,
$$

where $\Delta_{h, \delta} f(x, y)=f(x+h, y+\delta)-f(x, y)$. Also we denote by $C_{\omega_{p, q}}^{m}$ is the set of all functions $f \in C_{\omega_{p, q}}$ with the partial derivatives $f_{x^{j}, y^{k-j}}^{(k)}, k=1,2, \ldots, m$, belonging to $C_{\omega_{p, q}}$.

Let $\left\{\varphi_{n}\right\}(n=1,2, \ldots)$ is a sequence of functions $\varphi_{n}: \mathbb{C} \rightarrow \mathbb{C}$ satisfying the following properties: 
(i) $\varphi_{n} \quad(n=1,2, \ldots)$ is analytic on a domain $D$ containing the disk $B=\{z \in C:|z-b| \leq b\} \subset D$;

(ii) $\varphi_{n}(0)=1(n=1,2, \ldots)$;

(ii i ) for any $x \geq 0, \varphi_{n}(x)>0$ and $\varphi_{n}^{(k)}(0) \geq 0$ for any $n=1,2, \ldots$ and $k=1,2, \ldots$.

(iv) for every $n=1,2, \ldots$

$$
\frac{\varphi_{n}^{(v)}\left(a_{n} x\right)}{n^{v} \varphi_{n}\left(a_{n} x\right)}=1+o\left(\frac{1}{b_{n}}\right) \quad v=0,1,2,3,4
$$

where $a_{n}=\frac{b_{n}}{n} \rightarrow 0$ and $b_{n} \rightarrow \infty$ as $n \rightarrow \infty$.

For a real valued function $f$ defined on the interval $[0, \infty)$, generalized Balazs type operator defined by (see [8])

$$
L_{n}(f ; x)=\frac{1}{\varphi_{n}\left(a_{n} x\right)} \sum_{k=0}^{\infty} f\left(\frac{k}{b_{n}}\right) \frac{\varphi_{n}^{(k)}(0)}{k !}\left(a_{n} x\right)^{k} \quad(n=1,2, \ldots) .
$$

In (1.2), choosing $a_{n}=1$ we obtain following operators

$$
L_{n}(f ; x)=\frac{1}{\varphi_{n}(x)} \sum_{k=0}^{\infty} f\left(\frac{k}{b_{n}}\right) \frac{\varphi_{n}^{(k)}(0)}{k !} x^{k}
$$

which are known as the Baskakov type operator. It can be easily verified that in case $\varphi_{n}(x)=(1+x)^{n}$, the operators $L_{n}(f ; x)$ reduce to the well known Bernstein type rational function introduced by $\mathrm{K}$. Balazs[3] as

$$
L_{n}(f ; x)=\frac{1}{\left(1+a_{n} x\right)^{n}} \sum_{k=0}^{n}\left(\begin{array}{l}
n \\
k
\end{array}\right)\left(a_{n} x\right)^{k} f\left(\frac{k}{b_{n}}\right) .
$$

In the present work, inspired by operator (1.2), for $f \in C_{\omega_{p, q}}$ and $p, q \in \mathbb{N}_{0}$ we introduce the following operators

$$
L_{n, m}\left(f ; a_{n}, b_{n}, c_{m}, d_{m} ; x, y\right) \equiv L_{n, m}(f ; x, y)
$$

defined by

$$
=\frac{1}{\varphi_{n}\left(a_{n} x\right)} \frac{1}{\varphi_{m}\left(c_{m} y\right)} \sum_{j=0}^{\infty} \sum_{k=0}^{\infty} \frac{\varphi_{n}^{(j)}(0)}{j !}\left(a_{n} x\right)^{j} \frac{\varphi_{m}^{(k)}(0)}{k !}\left(c_{m} y\right)^{k} f\left(\frac{j}{b_{n}}, \frac{k}{d_{m}}\right),
$$

$$
(x, y) \in \mathbb{R}_{0}^{2}, n, m \in \mathbb{N}
$$


where $\left(a_{n}\right),\left(b_{n}\right),\left(c_{m}\right),\left(d_{m}\right)$ are given increasing and unbounded sequences of positive numbers such that

$$
\frac{\varphi_{n}^{(v)}\left(a_{n} x\right)}{n^{v} \varphi_{n}\left(a_{n} x\right)}=1+o\left(\frac{1}{b_{n}}\right), \frac{\varphi_{m}^{(v)}\left(c_{m} x\right)}{m^{v} \varphi_{m}\left(c_{m} x\right)}=1+o\left(\frac{1}{d_{m}}\right) .
$$

From (1.3) and (1.4) we decide that $L_{n, m}(f)$ are well-defined in every space $f \in C_{\omega_{p, q}}$ and $p, q \in \mathbb{N}_{0}$. Moreover, for $(x, y) \in \mathbb{R}_{0}^{2}, n, m \in \mathbb{N}$, we have

$$
L_{n, m}\left(1 ; a_{n}, b_{n}, c_{m}, d_{m} ; x, y\right)=1 \text {. }
$$

If $f \in C_{\omega_{p, q}}$ and $f(x, y)=f_{1}(x) f_{2}(y)$; for all $(x, y) \in \mathbb{R}_{0}^{2}$ and $n, m \in \mathbb{N}$ then

$$
L_{n, m}\left(f ; a_{n}, b_{n}, c_{m}, d_{m} ; x, y\right)=L_{n}\left(f_{1} ; a_{n}, b_{n} ; x\right) L_{m}\left(f_{2} ; c_{m}, d_{m} ; y\right) \text {. }
$$

This paper is devoted to a study aimed at obtaining approximation results by using the modulus of continuity and obtaining a Voronovskaya type theorem for the Baskakov type operators defined by (1.3) in polynomial weighted spaces. Approximation results for some different operators in the weighted spaces have been investigated in some papers (e.g. [2],[6], [5], [7],[4], [8], [9],[10], [12], [11]).

\section{Auxiliary Results}

In this section, we give some lemmas, which are essential to prove our main theorems.

Lemma 1. Let $L_{n}(f ; x)$ be defined by (1.2). For $x \in \mathbb{R}_{0}$ and $n \in \mathbb{N}$, we have

$$
\begin{gathered}
L_{n}(1 ; x, y)=1, \\
L_{n}(t-x ; x, y)=\left(\frac{a_{n}}{b_{n}} \frac{\varphi_{n}^{\prime}\left(a_{n} x\right)}{\varphi_{n}\left(a_{n} x\right)}-1\right) x, \\
L_{n}\left((t-x)^{2} ; x\right)=\left(\frac{a_{n}^{2}}{b_{n}^{2}} \frac{\varphi_{n}^{\prime \prime}\left(a_{n} x\right)}{\varphi_{n}\left(a_{n} x\right)}-2 \frac{a_{n}}{b_{n}} \frac{\varphi_{n}^{\prime}\left(a_{n} x\right)}{\varphi_{n}\left(a_{n} x\right)}+1\right) x^{2}+\frac{a_{n} x}{b_{n}^{2}} \frac{\varphi_{n}^{\prime}\left(a_{n} x\right)}{\varphi_{n}\left(a_{n} x\right)} \\
\text { where } a_{n}=\frac{b_{n}}{n} \rightarrow 0, b_{n} \rightarrow \infty \text { and } \frac{\varphi_{n}^{(v)}\left(a_{n} x\right)}{n^{v} \varphi_{n}\left(a_{n} x\right)}=1+o\left(\frac{1}{b_{n}}\right) .
\end{gathered}
$$

Lemma 2. For every fixed $p \in \mathbb{N}_{0}$, for all $x \in \mathbb{R}_{0}$ and $n \in \mathbb{N}$, we have

$$
\begin{gathered}
\omega_{p}(x) L_{n}\left(\frac{1}{\omega_{p}(t)} ; x\right) \leq M_{1}, \\
\omega_{p}(x) L_{n}\left(\frac{(t-x)^{2}}{\omega_{p}(t)} ; x\right) \leq M_{2}\left[\left(\frac{a_{n}^{2}}{b_{n}^{2}} \frac{\varphi_{n}^{\prime \prime}\left(a_{n} x\right)}{\varphi_{n}\left(a_{n} x\right)}-2 \frac{a_{n}}{b_{n}} \frac{\varphi_{n}^{\prime}\left(a_{n} x\right)}{\varphi_{n}\left(a_{n} x\right)}+1\right) x^{2}\right.
\end{gathered}
$$




$$
\left.+\frac{a_{n} x}{b_{n}^{2}} \frac{\varphi_{n}^{\prime}\left(a_{n} x\right)}{\varphi_{n}\left(a_{n} x\right)}\right]
$$

where $M_{1}, M_{2}$ are positive constants.

Lemma 3. For every $x \in \mathbb{R}_{0}$, one has

$$
\begin{gathered}
\lim _{n \rightarrow \infty} b_{n} L_{n}\left((t-x)^{k} ; x\right)=\left\{\begin{array}{cc}
0, & \text { if } k=1 \\
x, & \text { if } k=2
\end{array}\right. \\
\lim _{n \rightarrow \infty} b_{n}^{2} L_{n}\left((t-x)^{k} ; x\right)=\left\{\begin{array}{cc}
x, & \text { if } k=3 \\
3 x^{2}, & \text { if } k=4
\end{array} .\right.
\end{gathered}
$$

Lemma 4. For every $p, q \in \mathbb{N}_{0}, m, n \in \mathbb{N}$ and every $f \in C_{\omega_{p, q}}$, we have

where $M_{4}$ is a positive constant.

$$
\begin{gathered}
\left\|L_{n, m}\left(\frac{1}{\omega_{p, q}(t, z)}\right)\right\|_{C_{\omega_{p, q}}} \leq M_{4}, \\
\left\|L_{n, m}(f)\right\|_{C_{\omega_{p, q}}} \leq M_{4}\|f\|_{C_{\omega_{p, q}}}
\end{gathered}
$$

Lemma 5. Let $f \in C_{\omega_{p, q}}$ and $p, q \in \mathbb{N}_{0}$. For all $m, n \in \mathbb{N}$, we get

$$
\begin{aligned}
&\left\|\left(L_{n, m} f\right)_{x}^{\prime}\right\|_{C_{\omega_{p, q}}} \leq M_{5}\|f\|_{C_{\omega_{p, q}}} a_{n}, \\
&\left\|\left(L_{n, m} f\right)_{y}^{\prime}\right\|_{C_{\omega_{p, q}}} \leq M_{5}\|f\|_{C_{\omega_{p, q}}} c_{m}
\end{aligned}
$$

where $M_{5}$ is a positive constant.

\section{MAIN RESULTS}

Now, we give firstly following two theorems on the degree of approximation of functions by $L_{n, m}$ defined by (1.3).

Theorem 1. Let $f \in C_{\omega_{p, q}}^{1}$ and fixed $p, q \in \mathbb{N}_{0}$. For all $(x, y) \in \mathbb{R}_{0}^{2}$ and $m, n \in \mathbb{N}$, we have

$$
\omega_{p, q}(x, y)\left|L_{n, m}(f ; x, y)-f(x, y)\right| \leq M_{6}\left\{\left\|f_{x}^{\prime}\right\|_{p, q} \sqrt{\frac{x}{b_{n}}}+\left\|f_{y}^{\prime}\right\|_{p, q} \sqrt{\frac{y}{d_{m}}}\right\}
$$

where $M_{6}$ is a positive constant.

Proof of Theorem 1. Let $(x, y) \in \mathbb{R}_{0}^{2}$ be a fixed point. Then $f \in C_{\omega_{p, q}}^{1}$ we have the formula

$$
f(t, z)-f(x, y)=\int_{x}^{t} f_{u}^{\prime}(u, z) d u+\int_{y}^{z} f_{v}^{\prime}(x, v) d v \quad(t, z) \in \mathbb{R}_{0}^{2} .
$$


Thus, by (1.5), we obtain

$$
\begin{gathered}
L_{n, m}(f(t, z) ; x, y)-f(x, y) \\
=L_{n, m}\left(\int_{x}^{t} f_{u}^{\prime}(u, z) d u ; x, y\right)+L_{n, m}\left(\int_{y}^{z} f_{v}^{\prime}(x, v) d v ; x, y\right)
\end{gathered}
$$

On the other hand, we have

$$
\begin{aligned}
\left|\int_{x}^{t} f_{u}^{\prime}(u, z) d u\right| & =\left|\int_{x}^{t} \omega_{p, q}(u, z) f_{u}^{\prime}(u, z) \frac{1}{\omega_{p, q}(u, z)} d u\right| \\
& \leq\left\|f_{x}^{\prime}\right\|_{p, q}\left\|\int_{x}^{t} \frac{1}{\omega_{p, q}(u, z)} d u\right\| \\
& \leq\left\|f_{x}^{\prime}\right\|_{p, q}\left(\frac{1}{\omega_{p, q}(t, z)}+\frac{1}{\omega_{p, q}(x, z)}\right)|t-x|,
\end{aligned}
$$

which implies (1.2) and (1.5) that

$$
\begin{gathered}
\omega_{p, q}(x, y)\left|L_{n, m}\left(\int_{x}^{t} f_{u}^{\prime}(u, z) d u ; x, y\right)\right| \\
\leq \omega_{p, q}(x, y) L_{n, m}\left(\left|\int_{x}^{t} f_{u}^{\prime}(u, z) d u\right| ; x, y\right) \\
\leq\left\|f_{x}^{\prime}\right\|_{p, q} \omega_{p, q}(x, y)\left\{L_{n, m}\left(\frac{|t-x|}{\omega_{p, q}(t, z)} ; x, y\right)+L_{n, m}\left(\frac{|t-x|}{\omega_{p, q}(x, z)} ; x, y\right)\right\} \\
\leq\left\|f_{x}^{\prime}\right\|_{p, q} \omega_{q}(y) L_{m}\left(\frac{1}{\omega_{q}(z)} ; y\right)\left\{\omega_{p}(x) L_{n}\left(\frac{|t-x|}{\omega_{p}(t)} ; x\right)+L_{n}(|t-x| ; x)\right\} .
\end{gathered}
$$

Applying the Hölder inequality and (2.1), (2.2), (2.3), we get the inequalities

$$
\begin{gathered}
L_{n}(|t-x| ; x) \leq\left\{L_{n}\left((t-x)^{2} ; x\right) L_{n}(1 ; x)\right\}^{1 / 2} \\
=\left\{\left(\frac{a_{n}^{2}}{b_{n}^{2}} \frac{\varphi_{n}^{\prime \prime}\left(a_{n} x\right)}{\varphi_{n}\left(a_{n} x\right)}-2 \frac{a_{n}}{b_{n}} \frac{\varphi_{n}^{\prime}\left(a_{n} x\right)}{\varphi_{n}\left(a_{n} x\right)}+1\right) x^{2}+\frac{a_{n} x}{b_{n}^{2}} \frac{\varphi_{n}^{\prime}\left(a_{n} x\right)}{\varphi_{n}\left(a_{n} x\right)}\right\}^{1 / 2} \\
=\left\{\left(\left(1+o\left(\frac{1}{b_{n}}\right)\right)-2\left(1+o\left(\frac{1}{b_{n}}\right)\right)+1\right) x^{2}+\frac{x}{b_{n}}\left(1+o\left(\frac{1}{b_{n}}\right)\right)\right\}^{1 / 2}
\end{gathered}
$$

for a sufficiently large $n$, we have

$$
L_{n}(|t-x| ; x) \leq M_{7} \sqrt{\frac{x}{b_{n}}},
$$

and so

$$
\omega_{p}(x) L_{n}\left(\frac{|t-x|}{\omega_{p}(t)} ; x\right)
$$




$$
\begin{gathered}
\leq\left\{\omega_{p}(x) L_{n}\left(\frac{(t-x)^{2}}{\omega_{p}(t)} ; x\right)\right\}^{1 / 2}\left\{\omega_{p}(x) L_{n}\left(\frac{1}{\omega_{p}(t)} ; x\right)\right\}^{1 / 2} \\
\leq M_{8} \sqrt{\frac{x}{b_{n}}} .
\end{gathered}
$$

Consequently

$$
\begin{gathered}
\omega_{p, q}(x, y)\left|L_{n, m}\left(\int_{x}^{t} f_{u}^{\prime}(u, z) d u ; x, y\right)\right| \leq M_{9}\left\|f_{x}^{\prime}\right\|_{p, q} \sqrt{\frac{x}{b_{n}}}, \\
\omega_{p, q}(x, y)\left|L_{n, m}\left(\int_{y}^{z} f_{v}^{\prime}(x, v) d v ; x, y\right)\right| \leq M_{10}\left\|f_{y}^{\prime}\right\|_{p, q} \sqrt{\frac{y}{d_{m}}} .
\end{gathered}
$$

Finally, the last two inequalities, for all $m, n \in \mathbb{N}$, we derive from (3),

$$
\omega_{p, q}(x, y)\left|L_{n, m}(f ; x, y)-f(x, y)\right| \leq M_{6}\left\{\left\|f_{x}^{\prime}\right\|_{p, q} \sqrt{\frac{x}{b_{n}}}+\left\|f_{y}^{\prime}\right\|_{p, q} \sqrt{\frac{y}{d_{m}}}\right\}
$$

Thus the proof of the theorem is completed.

Theorem 2. Let $f \in C_{\omega_{p, q}}$ and $p, q \in \mathbb{N}_{0}$. For all $(x, y) \in \mathbb{R}_{0}^{2}$ and $m, n \in \mathbb{N}$, we have

$$
\omega_{p, q}(x, y)\left|L_{n, m}(f ; x, y)-f(x, y)\right| \leq M_{13} \Omega\left(f ; \sqrt{\frac{x}{b_{n}}}, \sqrt{\frac{y}{d_{m}}}\right)
$$

where $M_{13}$ is a positive constant and $\Omega$ is the modulus of continuity of $f$.

Proof of Theorem 2. For $f \in C_{\omega_{p, q}}$, applying the Stieklov function $f_{h, \delta}$

$$
f_{h, \delta}=\frac{1}{h \delta} \int_{0}^{h} d u \int_{0}^{\delta} f(x+u, y+v) d v, \quad(x, y) \in \mathbb{R}_{0}^{2}, \quad h, \delta>0 .
$$

From (3.4), we can write

$$
f_{h, \delta}(x, y)-f(x, y)=\frac{1}{h \delta} \int_{0}^{h} d u \int_{0}^{\delta} \Delta_{u, v} f(x, y) d v
$$

and therefore

$$
\begin{gathered}
\left(f_{h, \delta}\right)_{x}^{\prime}(x, y)=\frac{1}{h \delta} \int_{0}^{h} d u \int_{0}^{\delta} \frac{\partial f}{\partial u}(x+u, y+v) d v \\
=\frac{1}{h \delta} \int_{0}^{\delta}\{(f(x+h, y+v)-f(x, y))-(f(x, y+v)-f(x, y))\} d v \\
=\frac{1}{h \delta} \int_{0}^{\delta}\left(\Delta_{h, v} f(x, y)-\Delta_{0, v} f(x, y)\right) d v
\end{gathered}
$$


Similarly, we can obtain

$$
\left(f_{h, \delta}\right)_{y}^{\prime}(x, y)=\frac{1}{h \delta} \int_{0}^{h}\left(\Delta_{u, \delta} f(x, y)-\Delta_{u, 0} f(x, y)\right) d u .
$$

Thus we have

$$
\begin{aligned}
\left\|f_{h, \delta}-f\right\|_{C_{\omega_{p, q}}} & =\sup \omega_{p, q}(x, y)\left|f_{h, \delta}(x, y)-f(x, y)\right| \\
& =\sup \omega_{p, q}(x, y)\left|\frac{1}{h \delta} \int_{0}^{h} d u \int_{0}^{\delta} \Delta_{u, v} f(x, y) d v\right| \\
& \leq \Omega(f ; h, \delta),
\end{aligned}
$$

and from (3.5) and (3.6), we get

$$
\begin{aligned}
\left\|\left(f_{h, \delta}\right)_{x}^{\prime}\right\|_{C_{\omega_{p, q}}} & \leq \frac{2}{h} \Omega(f ; h, \delta), \\
\left\|\left(f_{h, \delta}\right)_{y}^{\prime}\right\|_{C_{\omega_{p, q}}} & \leq \frac{2}{\delta} \Omega(f ; h, \delta) .
\end{aligned}
$$

Now, for $L_{n, m}$ defined by (1.3), we can write

$$
\begin{gathered}
\omega_{p, q}(x, y)\left|L_{n, m}(f ; x, y)-f(x, y)\right| \\
\leq \omega_{p, q}(x, y)\left\{\left|L_{n, m}\left(f(t, z)-f_{h, \delta}(t, z) ; x, y\right)\right|\right. \\
+\left|L_{n, m}\left(f_{h, \delta}(t, z) ; x, y\right)-f_{h, \delta}(x, y)\right|+\left|f_{h, \delta}(x, y)-f(x, y)\right| \\
=\lambda_{1}+\lambda_{2}+\lambda_{3} .
\end{gathered}
$$

Firstly, we consider $\lambda_{1}=\omega_{p, q}(x, y)\left|L_{n, m}\left(f(t, z)-f_{h, \delta}(t, z) ; x, y\right)\right|$. By (2.6) and (3.7) we have

$$
\begin{aligned}
\lambda_{1} & \leq\left\|L_{m, n}\left(f-f_{h, \delta}\right)\right\|_{C_{\omega_{p, q}}} \\
& \leq M_{4}\left\|f-f_{h, \delta}\right\|_{C_{\omega_{p, q}}} \\
& \leq M_{4} \Omega(f ; h, \delta),
\end{aligned}
$$

and for $\lambda_{3}$, we get

$$
\begin{aligned}
\lambda_{3} & =\omega_{p, q}(x, y)\left|f_{h, \delta}(x, y)-f(x, y)\right| \\
& \leq\left\|f_{h, \delta}-f\right\|_{\omega_{\omega_{p, q}}} \\
& \leq \Omega(f ; h, \delta) .
\end{aligned}
$$

Finally, for $\lambda_{2}$, Applying Theorem 1 and (3.8) and (3.9), we obtain

$$
\begin{aligned}
\lambda_{2} & =\omega_{p, q}(x, y)\left|L_{n, m}\left(f_{h, \delta}(t, z) ; x, y\right)-f_{h, \delta}(x, y)\right| \\
& \leq M_{11}\left\{\left\|\left(f_{h, \delta}\right)_{x}^{\prime}\right\|_{\boldsymbol{C}_{\omega_{p, q}}} \sqrt{\frac{x}{b_{n}}}+\left\|\left(f_{h, \delta}\right)_{y}^{\prime}\right\|_{\boldsymbol{C}_{\omega_{p, q}}} \sqrt{\frac{y}{d_{m}}}\right\}
\end{aligned}
$$




$$
\begin{aligned}
& \leq M_{11}\left\{\frac{2}{h} \Omega(f ; h, \delta) \sqrt{\frac{x}{b_{n}}}+\frac{2}{\delta} \Omega(f ; h, \delta) \sqrt{\frac{y}{d_{m}}}\right\} \\
& \leq \frac{2}{M_{11}} \Omega(f ; h, \delta)\left\{\frac{1}{h} \sqrt{\frac{x}{b_{n}}}+\frac{1}{\delta} \sqrt{\frac{y}{d_{m}}}\right\} .
\end{aligned}
$$

Consequently, for $(x, y) \in \mathbb{R}_{0}^{2}, m, n \in \mathbb{N}$ and $h, \delta>0$, there exists $M_{13}$ we get

$$
\omega_{p, q}(x, y)\left|L_{n, m}(f ; x, y)-f(x, y)\right| \leq M_{13} \Omega(f ; h, \delta)\left\{1+\frac{1}{h} \sqrt{\frac{x}{b_{n}}}+\frac{1}{\delta} \sqrt{\frac{y}{d_{m}}}\right\} .
$$
result.

For fixed $x, y>0$, taking $h=\sqrt{\frac{x}{b_{n}}}$ and $\delta=\sqrt{\frac{y}{d_{m}}}$ in (3.10), we obtain desired

From Theorem 2, we obtain following approximation theorem for $L_{n, m}$ operators defined by (1.3):

Theorem 3. Let $f \in C_{\omega_{p, q}}$ and $p, q \in \mathbb{N}_{0}$. Then

$$
\lim _{n, m \rightarrow \infty} L_{n, m}(f ; x, y)=f(x, y) \quad \text { for all }(x, y) \in \mathbb{R}_{0}^{2} .
$$

Moreover (3.11) holds uniformly on every rectangle $0 \leq x \leq x_{0}, \quad 0 \leq y \leq y_{0}$ (see Example 1).

Remark 1. Some particular cases of the operators (1.3) are defined as follows: If $\varphi_{n}(x)=e^{a_{n} x}, \varphi_{m}(y)=e^{c_{m} y}$, then the operators $L_{n, m}(f ; x, y)$ reduce to the Szász type operators of two variables defined by

$$
\begin{aligned}
L_{n, m}(f ; x, y) & =S_{n, m}(f ; x, y) \\
& =e^{-\left(a_{n} x+c_{m} y\right)} \sum_{k=0}^{\infty} \sum_{j=0}^{\infty} \frac{\left(a_{n} x\right)^{k}}{k !} \frac{\left(c_{m} y\right)^{j}}{j !} f\left(\frac{k}{b_{n}}, \frac{j}{d_{m}}\right),(x, y) \in \mathbb{R}_{0}^{2} .
\end{aligned}
$$

If $\varphi_{n}(x)=(1+x)^{n}, \varphi_{m}(y)=(1+y)^{m}$, then the operators $L_{n, m}(f ; x, y)$ reduce to Bernstein Balazs type operators of two variables defined by

$$
\begin{gathered}
L_{n, m}(f ; x, y)=R_{n, m}(f ; x, y) \\
=\frac{1}{\left(1+a_{n} x\right)^{n}} \frac{1}{\left(1+c_{m} y\right)^{m}} \sum_{k=0}^{n} \sum_{j=0}^{m}\left(\begin{array}{c}
n \\
k
\end{array}\right)\left(\begin{array}{c}
m \\
j
\end{array}\right)\left(a_{n} x\right)^{k}\left(c_{m} y\right)^{j} f\left(\frac{k}{b_{n}}, \frac{j}{d_{m}}\right), \\
(x, y) \in \mathbb{R}_{0}^{2} .
\end{gathered}
$$

Example 1. For $\varphi_{n}(x)=e^{a_{n} x}, \varphi_{m}(y)=e^{c_{m} y}$ and $a_{n}=\sqrt{n}+1, b_{n}=\sqrt{n} ; c_{m}=$ $\sqrt{m}+1, d_{m}=\sqrt{m}$; the convergence of $L_{n, m}(f ; x, y)=S_{n, m}(f ; x, y)$ to $f(x, y)=$ $x y e^{-(x+y)}$ will be illustrated following Fig.(1). 


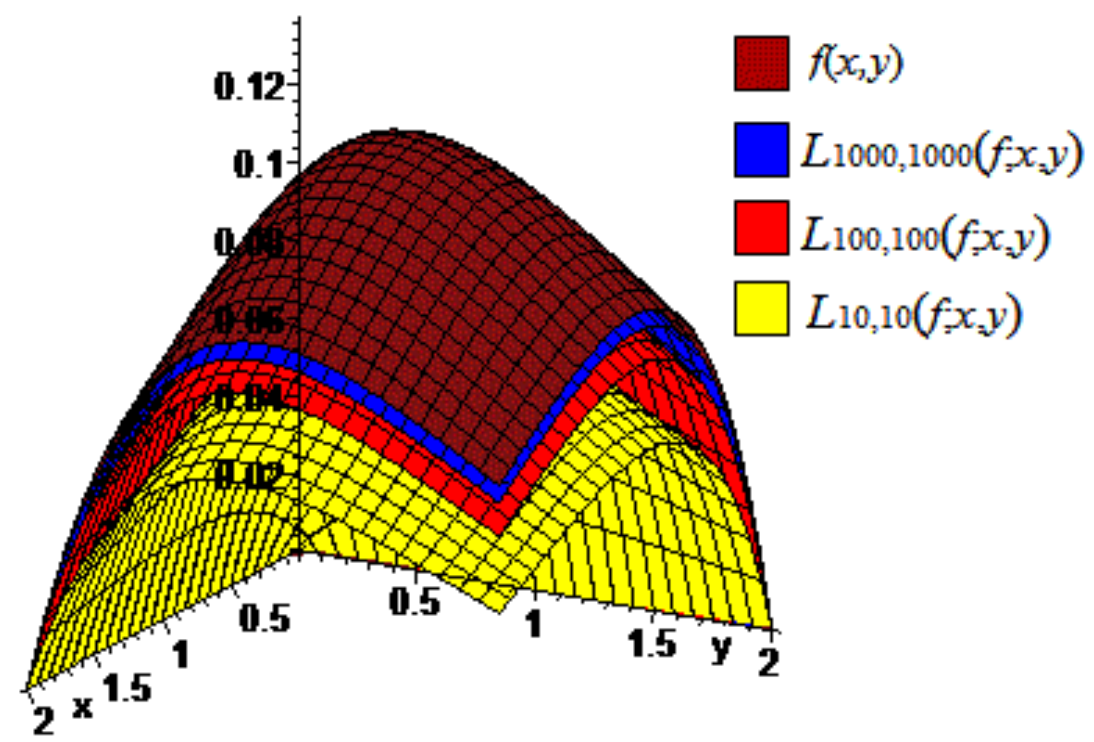

FIGURE 1. Convergenge of the $L_{n, m}=S_{n, m}$ operators for $n, m=10,100,1000$.

Example 2. For $a_{n}=\sqrt{n}+1, b_{n}=\sqrt{n} ; c_{m}=\sqrt{m}+1, d_{m}=\sqrt{m}$; the convergence of $S_{n, m}(f ; x, y)$ to $f(x, y)=\frac{x}{1+x^{2}} e^{-y}$ and the convergence of $R_{n, m}(f ; x, y)$ to $f(x, y)=\frac{x}{1+x^{2}} e^{-y}$ will be illustrated following Fig.(2).

Now, we give the Voronovskaya type theorem for the following operators given by (1.3) for $n=m$ :

$$
=\frac{1}{\varphi_{n}\left(a_{n} x\right)} \frac{1}{\varphi_{n}\left(c_{n} y\right)} \sum_{j=0}^{\infty} \sum_{k=0}^{\infty} \frac{\varphi_{n}^{(j)}(0)}{j !}\left(a_{n} x\right)^{j} \frac{\varphi_{n}^{(k)}(0)}{k !}\left(c_{n} y\right)^{k} f\left(\frac{j}{b_{n}}, \frac{k}{b_{n}}\right),
$$

$(x, y) \in \mathbb{R}_{0}^{2}, n \in \mathbb{N}$, where $a_{n}=\frac{b_{n}}{n} \rightarrow 0$ and $b_{n} \rightarrow \infty$ and $\frac{\varphi_{n}^{(v)}\left(a_{n} x\right)}{n^{v} \varphi_{n}\left(a_{n} x\right)}=1+$ $o\left(\frac{1}{b_{n}}\right)$.

Theorem 4. Let $f \in C_{\omega_{p, q}}^{2}$ and $p, q \in \mathbb{N}_{0}$. Then for every $(x, y) \in \mathbb{R}_{+}^{2}=(0, \infty) \times$ $(0, \infty)$

$$
\lim _{n \rightarrow \infty} b_{n}\left\{L_{n, n}(f ; x, y)-f(x, y)\right\}=\frac{x}{2} f_{x x}^{\prime \prime}(x, y)+\frac{y}{2} f_{y y}^{\prime \prime}(x, y) .
$$




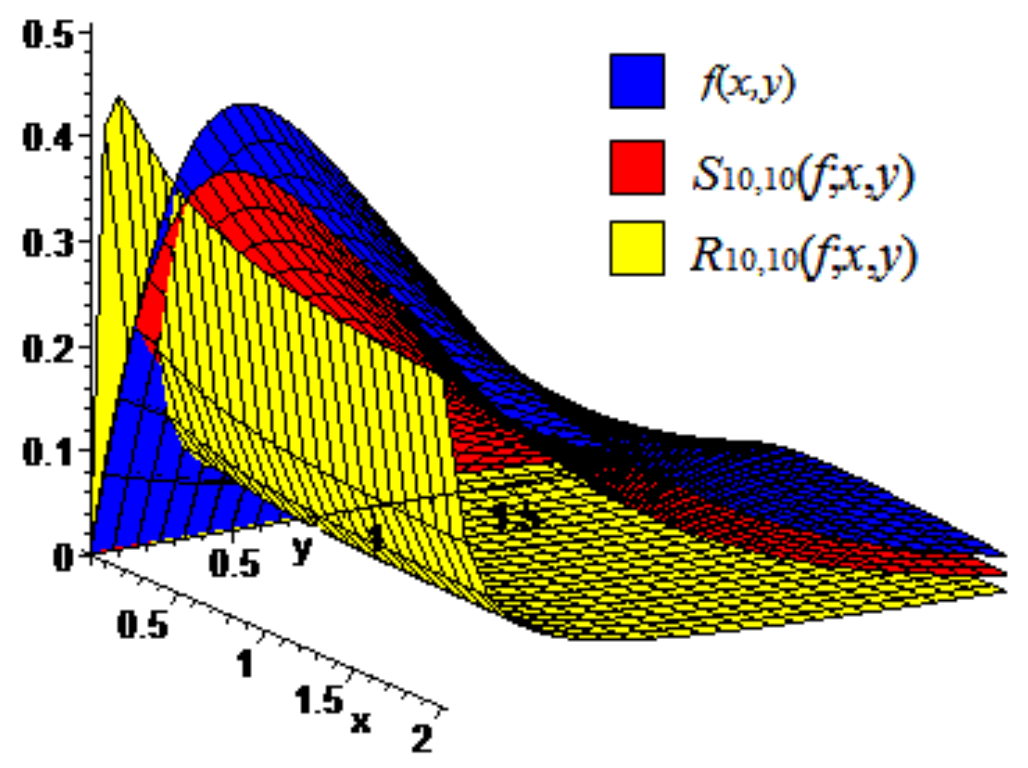

FIGURE 2. Convergenge comparation of the Szasz operators and Bernstein Balazs operators of two varibles for $n, m=10$.

Proof of Theorem 4. Choosing $(x, y) \in \mathbb{R}_{+}^{2}$, by the Taylor formula for $f \in C_{\omega_{p, q}}^{2}$, we have

$$
\begin{aligned}
f(t, z) & =f(x, y)+f_{x}^{\prime}(x, y)(t-x)+f_{y}^{\prime}(x, y)(z-y) \\
& +\frac{1}{2}\left\{f_{x x}^{\prime \prime}(x, y)(t-x)^{2}+2 f_{x y}^{\prime \prime}(x, y)(t-x)(z-y)+f_{y y}^{\prime \prime}(z-y)^{2}\right\} \\
& +\varepsilon_{1}(t, z) \sqrt{(t-x)^{4}+(z-y)^{4}}
\end{aligned}
$$

where $\varepsilon_{1}(t, z)=\varepsilon_{1}(t, z ; x, y)$ is a function belong to $C_{p, q}$ and $\varepsilon_{1}(x, y)=0$. From this and by (1.1), (1.3), (1.5)-(2.1) and (3.12), we can write the following equality

$$
\begin{gathered}
L_{n, n}(f(t, z) ; x, y) \\
=f(x, y)+f_{x}^{\prime}(x, y) L_{n}((t-x) ; x)+f_{y}^{\prime}(x, y) L_{n}((z-y) ; y) \\
+\frac{1}{2}\left\{f_{x x}^{\prime \prime}(x, y) L_{n}\left((t-x)^{2} ; x\right)+2 f_{x y}^{\prime \prime}(x, y) L_{n}((t-x) ; x) L_{n}((z-y) ; y)\right. \\
\left.+f_{y y}^{\prime \prime} L_{n}\left((z-y)^{2} ; y\right)\right\}+L_{n, n}\left(\varepsilon_{1}(t, z) \sqrt{(t-x)^{4}+(z-y)^{4}} ; x, y\right)
\end{gathered}
$$

by using (2.2), (2.3) and Lemma 2 , for $n \in \mathbb{N}$ we have

$$
\lim _{n \rightarrow \infty} b_{n}\left\{L_{n, n}(f ; x, y)-f(x, y)\right\}=\frac{x}{2} f_{x x}^{\prime \prime}(x, y)+\frac{y}{2} f_{y y}^{\prime \prime}(x, y)
$$




$$
+\lim _{n \rightarrow \infty} b_{n} L_{n, n}\left(\varepsilon_{1}(t, z) \sqrt{(t-x)^{4}+(z-y)^{4}} ; x, y\right) .
$$

in view of (1.1), (1.5)- (2.1) and using the Hölder inequality, we get

$$
\begin{aligned}
& \left|L_{n, n}\left(\varepsilon_{1}(t, z) \sqrt{(t-x)^{4}+(z-y)^{4}} ; x, y\right)\right| \\
& \leq\left\{L_{n, n}\left(\varepsilon_{1}^{2}(t, z) ; x, y\right)\right\}^{1 / 2}\left\{L_{n, n}\left((t-x)^{4}+(z-y)^{4} ; x, y\right)\right\}^{1 / 2} \\
& \leq\left\{L_{n, n}\left(\varepsilon_{1}^{2}(t, z) ; x, y\right)\right\}^{1 / 2}\left\{L_{n}\left((t-x)^{4} ; x\right)+L_{n}\left((z-y)^{4} ; y\right)\right\}^{1 / 2}
\end{aligned}
$$

From Theorem 3 , we obtain

$$
\lim _{n \rightarrow \infty} L_{n, n}\left(\varepsilon_{1}^{2}(t, z) ; x, y\right)=\varepsilon_{1}^{2}(x, y)=0,
$$

and considering (3.15), (3.16) and Lemma 3 , we get

$$
\lim _{n \rightarrow \infty} b_{n} L_{n, n}\left(\varepsilon_{1}(t, z) \sqrt{(t-x)^{4}+(z-y)^{4}} ; x, y\right)=0 .
$$

Using the equality (3.17) in (3.14), the proof is completed.

Remark 2. Recently many generalizations of well-known positive linear operators, based on $q$-integers were introduced and studied widely by several authors (we refer the reader to [1]). One can define and study $q$-analogue of the operators (1.3) .

\section{REFERENCES}

[1] A. Aral, V. Gupta, and R. Agrawal, Applications of q-Calculus in Operator Theory, ser. SpringerLink : Bücher. London: Springer, 2013.

[2] C. Atakut and N. Ispir, "On bernstein type rational functions of two variables," Math. Slovaca, vol. 54, no. 3, pp. 291-301, 2004.

[3] K. Balazs, "Approximation by bernstein type rational functions," Acta Math. Acad. Sci. Hungar, vol. 26, no. 1-2, pp. 123-134, 1975, doi: 10.1007/BF01895955.

[4] V. Gupta, "Different durrmeyer variants of baskakov operators," Topics in Mathematical Analysis and Applications, vol. 94, pp. 419-446, 2014, doi: 10.1007/978-3-319-06554-0¹7.

[5] V. Gupta and R. Agrawal, Convergence Estimates in Approximation Theory, ser. SpringerLink : Bücher. London: Springer International Publishing, 2014.

[6] V. Gupta and N. Ispir, "On the bézier variant of generalized kantorovich type balazs operators," Applied Mathematics Letters, vol. 18, no. 9, pp. 1053-1061, 2005, doi: 10.1016/j.aml.2004.11.002.

[7] N. Ispir and C. Atakut, "Approximation by modified szász-mirakjan operators on weighted spaces,” Proc. Indian Acad. Sci. (Math. Sci.), vol. 112, no. 4, pp. 571-578, 2002.

[8] N. Ispir and C. Atakut, "Approximation by generalized balazs type rational functions," Int. J. Comput. Numer. Anal., vol. 4, no. 3, pp. 297-316, 2003.

[9] L. Rempulska and S. Graczyk, "On generalized szász-mirakjan operators of functions of two variables," Math. Slovaca, vol. 62, no. 1, pp. 87-98, 2012.

[10] Z. Walczak, "On certain modified szász-mirakyan operators for functions of two variables," Demonstratio Math., vol. 33, no. 1, pp. 91-100, 2000. 
[11] Z. Walczak, "Approximation by some linear positive operators of functions of two variables," Saitama Math. J., vol. 21, pp. 23-31, 2003.

[12] Z. Walczak, "Baskokov type operators," Rocky Mountain Jourmal of Mathematics, vol. 39, no. 3, pp. 981-993, 2009, doi: 10.1216/RMJ-2009-39-3-981.

Authors' addresses

Çiğdem Atakut

Ankara University, Faculty of Science, Department of Mathematics,, Tandoğan, 06100 Ankara, Turkey

E-mail address: atakutescience. ankara.edu.tr

İbrahim Büyükyazici

Ankara University, Faculty of Science, Department of Mathematics,, Tandoğan, 06100 Ankara, Turkey

E-mail address: ibuyukyazici@gmail.com

Sevilay K. Serenbay

Başkent University, Department of Mathematics Education,, 06530 Ankara, Turkey

E-mail address: kirci@baskent.edu.tr 